\title{
The Intersection of Brownian Paths as a Case Study of a Renormalization Group Method for Quantum Field Theory
}

Michael Aizenman*

Departments of Mathematics and Physics, Rutgers University, New Brunswick, NJ 08903, USA

\begin{abstract}
A new approach is presented for the study of the probability that the random paths generated by two independent Brownian motions in $\mathbb{R}^{d}$ intersect or, more generally, are within a short distance $a$ of each other. The well known behavior of that function of $a$-above, below, and at the critical dimension $d=4$, as well as further corrections, are derived here by means of a single renormalization group equation. The equation's derivation is expected to shed some light on the $\beta$-function of the $\lambda \phi_{d}^{4}$ quantum field theory.
\end{abstract}

\section{Introduction}

The Brownian motion in addition to its own attraction has been a source of insight in various branches of mathematical analysis and physics. In particular, its properties have been quoted in the analysis of various "random walk" and "random surface", expansions used in statistical mechanics and quantum field theory. Since it is often the case that the insight is based on an analogy - rather than a complete reduction, the interest is not confined to the results, but extends also to the methods by which these can be proven. Thus we expect that something may still be gained from new derivations of old results (some of which are improved here).

With these comments in mind, we present here a new method for the study of the probabilities of intersection, and almost-intersection, for the paths of two independent Brownian motions: above, below and at the critical dimension $d=4$. In contrast with the previously available proofs, the analysis is based on a single equation - whose derivation requires only the more basic properties of the Bronian motion. In particular, a stronger use is made of the scale covariance - by means of which a " $\beta$-function" is derived for this problem.

* Sloan Foundation Research Fellow. Research supported in part by NSF grant PHY-8301493 
The above terminology is used in recognition of the similarity of the main equation with the one which appears in the Callan-Symanzik equations for the $\lambda \phi_{d}^{4}$ quantum field theory, where it is widely believed to describe Wilson's "renormalization group flow" of the coupling constant. While the equations are similar, their derivations are quite different. In fact, part of the motivation for this study is the desire to develop some insight which might be useful for a nonperturbative analysis of quantum-field theoretical and statistic-mechanical issues (see Sect. 8).

A relation between the Brownian motion and the more complex scalar quantum field theory, for which $d=4$ is the critical dimension, first appeared, more than a decade and a half ago, in the work of Professor K. Symanzik. He also discovered the renormalization group equation whose analog is considered here. Recent works on the subject, including this one, derive from, and were inspired by his deep insights - which we are only beginning to understand. I should like to present this work as a small tribute to Professor Symanzik's scientific contributions.

The main problem studied here, and the organization of the paper, are described in the next section.

\section{Brownian Motion}

With the term Brownian motion we refer to the stochastic process with continuous paths $b(t):[0, \infty) \rightarrow \mathbb{R}^{d}$, which is described by the Wiener measure. $b(t)$ has independent increments, and its transition probabilities are:

$$
\int \mu_{x}(d b) \delta(b(t)-y)=e^{t \Delta}(x, y)=(2 \pi t)^{-d / 2} e^{-(x-y)^{2} /(2 t)},
$$

where $\mu_{x}(d b)$ is probability measure for paths which start at $x \in \mathbb{R}^{d}$ (with $\int \mu_{x}(d b)=1$ ), and $\Delta$ is the Laplace operator. The measure $\mu$ defined by the above properties can be formally described by the following expression

$$
\mu_{x}(d b)=\delta(b(0)-x) \prod_{t \in[0, \infty)} d b(t) e^{-\frac{1}{2} \int\left|\frac{d b(s)}{d s}\right|^{2} d s} / \text { Normalization. }
$$

The realizability of the measure $\mu$ on the space of continuous functions is, of course, not a matter of definition - but a well known theorem. The Brownian motion has an alternative description as the continuum limit of a simple random walk (with a proper scaling).

For each $b(t)$ we denote by $\omega$ its "path" in $\mathbb{R}^{d}$. Specifically:

$$
\omega_{\left[t_{1}, t_{2}\right]}=b\left(\left[t_{1}, t_{2}\right]\right)=\left\{y \in \mathbb{R}^{d} \mid y=b(s) \text { for some } s \in\left[t_{1}, t_{2}\right]\right\},
$$

and $\omega=\omega_{[0, \infty)}$. From this point on, by the term path we refer only to the set $\omega$ (not $b(\cdot))$.

We shall discuss the behavior, in the limit $a \searrow 0$, of the probability that the paths of two independent Brownian motions are within the distance $a$ of each other, i.e.

$$
\begin{aligned}
& \operatorname{Prob}\left(\operatorname{dist}\left(\omega_{\left[0, t_{1}\right.}^{1}, \omega_{\left[0, t_{2}\right]}^{2}\right) \leqq a \mid 0, x\right) \\
& \stackrel{\text { def }}{=} \int \mu_{0}\left(d b^{1}\right) \mu_{x}\left(d b^{2}\right) \chi\left[\operatorname{dist}\left(\omega_{\left[0, t_{1}\right]}^{1}, \omega_{\left[0, t_{2}\right]}^{2}\right) \leqq a\right] .
\end{aligned}
$$


The symbol $\chi[\mathscr{C}]$ will always denote a characteristic function which is 1 if the condition $\mathscr{C}$ is satisfied, and 0 otherwise. ${ }^{1}$

Since the motion is continuous, the probability that the paths actually intersect is also well defined, and for finite $t_{i}$ it equals the limit of (2.4) as $a \searrow 0$.

The main intersection properties (but not all, see Sect. 8) of Brownian paths are known. The pioneering works of Kakutani [1] and Dvoretzky, Erdös, and Kakutani [2] showed the following.

Proposition 2.1 [1, 2]. For independent pairs of d-dimensional Brownian motions:

for each $x \neq 0$ and $t_{1}, t_{2}>0$.

$$
\operatorname{Prob}\left(\omega_{\left[0, t_{1}\right]}^{1} \cap \omega_{\left[0, t_{2}\right]}^{2} \neq \emptyset \mid 0, x\right)= \begin{cases}0 & d \geqq 4 \\ f\left(x, t_{1}, t_{2}\right) \neq 0 & d<4\end{cases}
$$

One may be puzzled by the fact that the critical dimension is $d=4$, since $\omega^{i}$ are the images, under the continuous maps $b^{i}$, of the one dimensional $\mathbb{R}^{+}$. Nevertheless, the Hausdorff dimensions of $\omega^{i}$ are ("almost surely") 2.

Each of the previously available proofs of (2.5) deals separately with the two cases distinguished there, and (except for $d>4$ ) employs some property of the Brownian motion which is a not quite trivial consequence of its definition. Our main purpose is to present a simple, and unified proof. As a bonus, we hope to shed some light on a renormalization-group equation which has appeared (by quite a different reasoning) in quantum field theory.

For simplicity we shall focus our attention on the single function,

$$
g(a)=\int_{\mathbb{R}^{d}} d x \int_{0}^{\infty} d t_{1} e^{-t_{1}} \int_{0}^{\infty} d t_{2} e^{-t_{2}} \operatorname{Prob}\left(\operatorname{dist}\left(\omega_{\left[0, t_{1}\right]}^{1}, \omega_{\left[0, t_{2}\right]}^{2}\right) \leqq a \mid 0, x\right),
$$

which can be shown to describe also the limiting behavior of the probability (2.4), as $a \rightarrow 0$, at each fixed $x \neq 0$ and $t_{1}, t_{2}>0$. [It is easy to see that $g(a)<\infty$; e.g. by the argument used in (6.13).]

The arguments presented here offer a simple proof that, as $a \rightarrow 0, g(a)$ exhibits the following behavior (in a strong sense - see Proposition 5.2):

$$
g(a) \cong\left\{\begin{array}{lr}
c_{d} \geqq C(4-d) & d<4, \\
C(d-4) a^{d-4}\left(1+0\left(a^{d-4+\min \{0,6-d\}}\right)\right) & d>4 \\
& \neq 6, \\
C(d-4) a^{d-4}\left(1+0\left(|\ln a| a^{d-4}\right)\right) & d=6
\end{array}\right.
$$

and, in the critical dimension $d=4$;

$$
\frac{c}{1+|\ln a|} \geqq g(a) \geqq \frac{c^{\prime}}{1+|\ln a|}
$$

Here, and in the rest of the paper $C$ stands for various constants, which we can bound from above and below. Furthermore, these bounds have natural extensions to non-integral values of $d$ - which are regular (i.e. neither vanish nor diverge for finite $d$ ).

1 In this work the superscipts on $b^{i}$ and $\omega^{i}$ should always be read as indices, not powers 
The leading behavior in (2.7) and (2.8) has in effect been known. Previous results, and methods, are briefly described in the next section. The emphasis in this paper is on the $\beta$-function formalism, which is introduced in Sect. 4. Our bounds on the $\beta$-function, $W(g)$, are summarized in Proposition 4.1. Their derivation is postponed to Sects. 6 and 7. In Sect. 5 the results on $W(g)$ are used to present a simple picture of the $d$-dependence of $g(0)$, and for the derivation of the above asymptotics for $g(a)$. Some open problems which call for an improvement and extensions of this approach are mentioned in Sect. 8.

Section 7 contains also some useful direct bounds on $g(a)$. Yet another point of view is described in the appendix.

\section{Previous Arguments}

The earliest analysis of the intersection properties of Brownian paths in $d=4$ and $d<4$ dimensions [2] was based on the fact that the probability of a $d$-dimensional Brownian motion to hit a set $A \subset \mathbb{R}^{d}$ does not vanish if and only if the sets' Newtonian capacity is non-zero. Furthermore, an additional basic - yet not entirely trivial - result of potential theory has been invoked for the case $d=3$; and for $d=4$ use was made of the somewhat sophisticated (yet by now standard) uniform estimate of Levy ${ }^{2}$

$$
|b(s)-b(t)| \leqq M(b, T) \sqrt{|t-s| \ln |t-s|^{-1}}, \quad s, t \in[0, T] .
$$

Closely related questions were considered in [3] with regard to intersection properties of discrete random walks (see Sect. 8). The stopping-time argument used there, led to an analog of the $|\ln a|^{-1}$ law for $d=4$ dimensions [see (2.8)]. An error of method (which led to incorrect results in other cases) was pointed out by Lawler [4], who proved this law by rather detailed considerations, combining stopping time arguments with another property which will not be described here.

Our quest for simpler methods is motivated by the desire to eventually be able to analyze the interaction in physical models which admit stochastic geometrical (interacting-) random walk or random surface representations, or expansions. Needless to say, for such models one does not yet have an analog of the potential theory, nor detailed information like (3.1).

The one case for which a simple argument has been known is the proof of the non-intersection of Brownian paths in $d>4$ dimensions (first derived in [1]). In Sect. 7 we recall a specially simple upper bound of Spencer [5] and derive, by a related method, a rather good lower bound for all $d$. An analog of Spencer's method was used for some of the results mentioned in 8 iii.

2 In fact, Levy's estimate can be used more effectively by considering the stopping time value of the function $f\left(b\left(t_{1}\right)\right)$, where

$$
f(z)=\int_{0}^{t_{1}} d t\left|z-b_{1}(t)\right|^{2-d}, \quad d=4
$$




\section{A Renormalization Group Equation}

\section{a) A Heuristic Argument}

A key idea in our approach to the study of the function $g(a)$ is to consider its changes under scaling. In particular, we shall make use of the Brownian motion's self similarity:

$$
b(t) \stackrel{\mathscr{Q}}{=} \alpha b\left(\alpha^{-2} t\right) \text { with respect to } \mu_{0}(d b),
$$

$\mathscr{D}$ meaning that the two functions have the same distribution. The well known scaling relation (4.1) is clearly seen in the formal expression (2.2), as well as in (2.1).

The scale covariance is, of course, broken by the constraint that $\operatorname{dist}\left(\omega_{\left[0, t_{2}\right]}^{1}\right.$, $\omega_{\left[0, t_{2}\right]}^{2} \leqq a$. Let us first offer a heuristic analysis of the resulting behavior of $g(a)$.

For an integer $n, g\left(\frac{a}{n}\right)$ may be related to $g(a)$ by the change of scale: $x=\hat{x} / n$, $t=\hat{t} / n^{2}$. When presented in terms of $\hat{x}$ and $\hat{t}$, in (4.1), $g\left(\frac{a}{n}\right)$ expresses the integral of the probability of pairs of longer paths to come within the distance $a$. The volume integral is $d x=n^{-d} d \hat{x}$, and the change in the time scale can be accounted for by replacing each path by a chain of $n^{2}$ linked paths - whose time duration is again of the order $0(1)$. The required contact would occur if in any of the $n^{2} \times n^{2}$ pairs the paths are within the distance $a$. Therefore, by the inclusion-exclusion principle:

$$
g\left(\frac{a}{n}\right)=n^{2 \cdot 2} n^{-d} g(a)-R(a, n)
$$

where $R$ is a (positive) multiple-intersection correction, which for a fixed $n$ we may expect it to behave as $O\left(g(a)^{2}\right)$.

Under iteration (4.2), with some more specific information on $R(a, n)$, leads to Theorem 1. We shall now present a more careful, and complete, argument in which (4.2) is replaced by a differential equation.

\section{b) The Main Result}

It is evident from (2.6) that $g(a)$ has the following properties

i) $g(a)$ is a strictly increasing function of $a$,

ii) $\lim _{a \rightarrow \infty} g(a)=\infty$,

iii) $\lim _{a \rightarrow 0} g(a)=g(0)$ (by the monotone convergence theorem).

The differentiability of the function $g(a)$ is certainly not manifest in (2.6). The situation may however be remedied by means of the scaling relation (4.1), with $\alpha=a$. One gets:

$$
\begin{aligned}
g(a) & =\int_{\mathbb{R}^{d}} d x \int_{0}^{\infty} d t_{1} d t_{2} e^{-\left(t_{1}+t_{2}\right)} \int \mu_{0}\left(d b^{1}\right) \mu_{x / a}\left(d b^{2}\right) \chi\left[\operatorname{dist}\left(\omega_{\left[0, t_{1} / a^{2}\right]}^{1}, \omega_{\left[0, t_{2} / a^{2}\right]}^{2}\right) \leqq 1\right] \\
& =a^{d+4} \int_{\mathbb{R}^{d}} d x \iint_{0}^{\infty} d t_{1} d t_{2} e^{-a^{2}\left(t_{1}+t_{2}\right)} \int \mu_{0}\left(d b^{1}\right) \mu_{x}\left(d b^{2}\right) \chi\left[\operatorname{dist}\left(\omega_{\left(0, t_{1}\right]}^{1}, \omega_{\left[0, t_{2}\right]}^{2}\right) \leqq 1\right] .
\end{aligned}
$$

Equation (4.3) shows that $g(a)$ is infinitely differentiable in $a \in(0, \infty)$, and allows one to obtain an expression for its derivative, which is given in Lemma 6.1, below. The above listed properties of $g(a)$ allow one to define implicitly the function $W(g)$, on $(g(0, \infty) \subset \mathbb{R}$, to which we refer in the next proposition. 
Proposition 4.1. The function $g(a)$ obeys the following differential equation

$$
\frac{d g}{d \ln a^{-1}}=-W(g(a))
$$

where $W$ (the " $\beta$-function") is a continuous function, defined uniquely in the regime $[g(0), \infty)$, having the form

$$
W(g)=(d-4) g+4 \hat{F}(g),
$$

with a non-linear term which, for $g \leqq g(1)$ in the lower bound, satisfies

$$
c_{+} g^{2} \leqq \hat{F}(g) \leqq c_{\delta} g^{1+\frac{2}{d+2+\delta}} \quad(\text { for any } \delta>0) \text {. }
$$

Furthermore, $F(a)=\hat{F}(g(a))$ (given explicitly by (6.2)) obeys the following bounds (for $a \leqq 1$ )

$$
\left\{\begin{array}{ll}
C|d-4|^{2} & d \neq 4 \\
c(1+|\ln a|)^{2} & d=4
\end{array} \leqq F(a) /\left[a^{2(d-4)+\min (0,6-d)}\right] \leqq \begin{cases}C|6-d|^{-1} & d \neq 6 \\
c(1+|\ln a|) & d=6 .\end{cases}\right.
$$

With the restriction of the lower bound to $g \approx g(0),(4.6)$ holds with:

$c_{+}(0)=\sqrt{\pi} /\left[(2 \sqrt{2 \pi})^{d} \Gamma\left(\frac{d+1}{2}\right)\right], \quad c_{\delta}=\left(\frac{(d+\delta)(d+2+\delta)}{2 \sqrt{2 \pi^{d}}} \Gamma\left(\frac{\delta}{2}\right)\right)^{\frac{2}{d+2+\delta}}$.

Summary of the Proof. The main results here are the bounds on the $\beta$-function. The exact expression for $W(g(a))$ is derived in Lemma 6.1 , where $F(a)$ is defined explicitly. The continuity of $W(g)$ at $g(0)$ follows from the obvious monotonicity of $F(a)$ [see (6.2)]. For the bounds (4.6) and (4.7) we use two different sets of arguments. (4.6) is proven by Lemmas 6.2 and 6.3; and (4.7) is derived, by direct estimates, in Sect. 7c.

Of the two inequalities in (4.6) the more faithful one is the lower bound. The other may in fact be improved using (4.7). However, (4.6) represents our a priori estimates which allow one to establish the main elements of the picture described next.

Let us precede the derivation of the results described above by a presentation of their implications.

\section{Analysis of the Renormalization-Group Inequalities}

The function $W(g)$ and a single value, say $g(1)$, contain all the information about $g(a)$ - the question of the vanishing of $g(0)$ being determined by $W(g)$ for $g \ll 1$. Notice that in (4.5) we give $W$ 's leading linear term exactly, and determine that the non-linear part is positive. In the role it plays, and its dependence on $g$ and the dimension $d$ (Fig. 1), $W$ is quite similar to the $\beta$-function of the $\phi_{d}^{4}$ euclidean-field [6], which was mentioned in the introduction. Its analysis belongs to the standard repertoire of physics literature (see, e.g. [7]).

For $g>g(0), W(g)$ is positive and, by (6.2), it converges to a limit as $g \searrow g(0)$. (4.4) shows that this limit has to be zero. For convenience, we shall refer to $W(\cdot)$ as if it is defined for all $g \in[0, \infty)$. Clearly, $W(g)$ has such extensions which have all the properties listed in Proposition 4.1. 


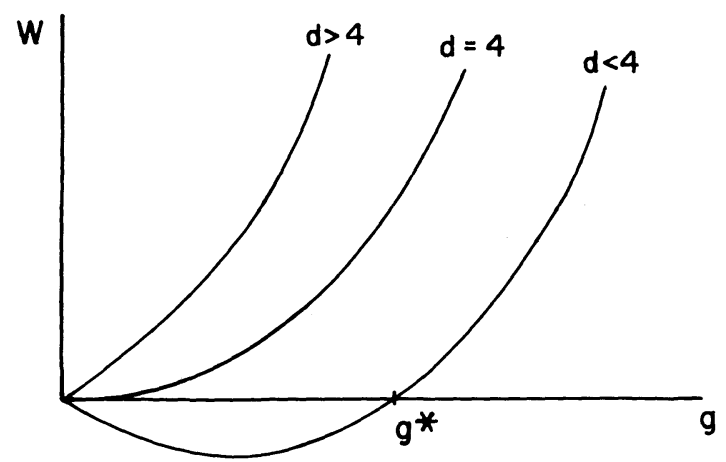

Fig. 1. The shape of the function $W(g)$

Proposition 5.1. In any dimension $d, g(a)$ takes values only in the region $\left[g^{*}, \infty\right)$, where

$$
g^{*}=\sup \{g \mid W(g)=0\}
$$

Furthermore, $g(0)=g^{*}$ and it satisfies:

$$
\left\{\begin{array}{lll}
g(0)=0 & \text { for } & d=4 \\
g(0)>\frac{2 \sqrt{2 \pi} d(4-d)^{(d+4) / 2}}{(d+2)(d+4)} & \text { for } & d<4 .
\end{array}\right.
$$

Remark. While (5.4) shows the main result for $d<4$, its $d$ dependence in the " $d \rightarrow 4$ limit" is not optimal, see the direct bound (7.12).

Proof. $g^{*}$ is well defined since $W$ is a continuous function, with

$$
W(0)=0 \text { and } \quad \lim _{g \rightarrow \infty} W(g)=\infty .
$$

The continuity of $g(a)$, and its properties i) and ii) (Sect. 4b) imply that $g(a) \in\left(g^{*}, \infty\right)$ for $a>0$, where $W(g)>0$.

As $a \rightarrow 0 g(a)$ which obeys Eq. (4.4), is driven all the way to $g^{*}$, since on the logarithmic scale of $\ln a^{-1}$, the interval $a \in(0,1]$ is mapped onto $[0, \infty)$.

The properties (5.2) and (5.3) of $g^{*}$ are simple consequences of (4.5) for which we choose $\delta=2$.

For dimensions $d \geqq 4$, where $g(0)$ vanishes, one is also interested in the actual behavior of $g(a)$ for small $a$.

Proposition 5.2. i) For dimensions $d>4$ :

$$
g(a)=c_{d} a^{d-4}\left(1+O\left(a^{\min (d-4,2)}\{1+|\ln a| \chi[d=6]\}\right)\right)
$$

in the strong sense that $0<c_{d} \leqq g(1)$ and the lower order term is also of not less than the indicated order, up to a logarithmic correction for $d=6$.

ii) In $d=4$ dimensions

$$
g(a) \leqq \frac{1}{g(1)+4 c_{+} \ln a^{-1}}
$$

for any $a \leqq 1$. (An opposite bound of the same order is provided by (7.12).) 
Proof. i) $d \geqq 4$. Notice that the simple bound

$$
\frac{d g}{d \ln a^{-1}} \leqq-(d-4) g
$$

[which is a consequence of the positivity of $\hat{F}(g)$, in (4.5)] implies that

$$
g(a) \leqq g(1) a^{d-4}, \quad \text { for } a \leqq 1 .
$$

For more complete information, let us write down the equation for $\hat{g}(a)$ $=g(a) / a^{d-4}$ :

$$
\frac{d \hat{g}(a)}{d \ln a^{-1}}=-F(a) a^{4-d}=-O\left(a^{\min (2, d-4)}\{1+|\ln a| \chi[d=6]\}\right)
$$

[by (4.5) and (4.7)]. Hence

$$
\hat{g}(a)=g(1)-\int_{0}^{\ln a^{-1}} d z O\left(e^{-\min (2, d-4) z}\{1+z \chi[d=6]\}\right) .
$$

Splitting

$$
\int_{0}^{\ln a^{-1}} d z \ldots=\int_{0}^{\infty} d z \ldots-\int_{\ln a^{-1}}^{\infty} d z \ldots
$$

we get

$$
g(a) / a^{d-4}=\mathrm{const}+O\left(a^{\min (2, d-4)}\{1+|\ln a| \chi[d=6]\}\right),
$$

which proves (5.5). Notice that the lower bound in (4.7) shows that the second term in the right-hand side is in fact of the indicated order of magnitude, up to logarithmic corrections for $d=6$ (and $d=4$ ).

ii) $d=4$. In this case $W(g)$ has no linear term, see (4.5). To integrate (4.6) we cast it in the following form:

$$
\frac{d g^{-1}}{d \ln a^{-1}} \geqq 4 c_{+} \quad \text { and } \frac{d g^{-\frac{1}{3+\delta}}}{d \ln a^{-1}} \leqq \frac{4}{3+\delta} c_{2 \delta},
$$

which directly leads to (5.6), and to a lower bound which looks like the right-hand side of (5.6) raised to the power $3+\delta$. [A better lower bound is given in (7.12).]

Remark. An alternative way to integrate bounds on $W(g)$ [but not those on $W(g(a))]$ is to invert and integrate (4.4), by which:

$$
\ln a^{-1}=\int_{g(a)}^{g(1)} d g W(g)^{-1} .
$$

This is the expression used in physics literature when one has a perturbative expansion for $W(g)$.

We have seen here how the problem introduced in Sect. 2 can be solved by the "renormalization group" equations (4.4) and (4.5). It still remains to prove the inequalities which lead to the bounds (4.6) and (4.7). This is done in the next two sections. 


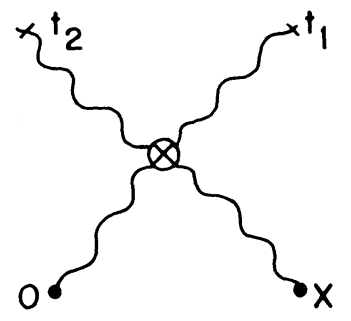

i) $g(a)$

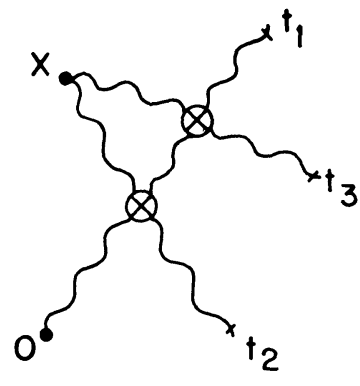

ii) $F(a)$

Fig. 2. A diagramatic representation of the functions $g(a)$ and $F(a)$. Each wiggled line represents a Brownian path, and a circle indicates that the paths come to within the distance $a . g$ and $F$ are obtained by integrating over $x$ and over the corresponding Brownian motions with an exponential killing time

\section{Proof of the Bounds on the $\beta$-Function}

a) An Exact Expression for $W(g(a))$

We start by differentiating the expression (4.3) for $g(a)$, producing in effect an exact version of the heuristic argument which led to (4.2).

Lemma 6.1. The function $g($ a) is infinitely differentiable in $(0, \infty)$, with the derivative

$$
a \frac{d g(a)}{d a}=(d-4) g(a)+4 F(a),
$$

where

$$
\begin{gathered}
F(a)=\int_{\mathbb{R}^{d}} d x \iint_{0}^{\infty} \int_{k=1}^{3}\left(d t_{k} e^{-t}\right) \int \mu_{0}\left(d b^{1}\right) \mu_{x}\left(d b^{2}\right) \mu_{x}\left(d b^{3}\right) \\
\cdot \chi\left[\operatorname{dist}\left(\omega_{\left[0, t_{1}\right]}^{1}, \omega_{\left[0, t_{i}\right.}^{i}\right) \leqq a ; i=2 \text { and } 3\right]
\end{gathered}
$$

(see Fig. 2).

Proof. The $C^{\infty}$ property follows directly from the expression (4.3) for $g(a)$. Differentiating (4.3) [which is symmetric in $\left(t_{1}, t_{2}\right)$ ], and then changing back to the scale of (2.6), we obtain:

with

$$
\frac{d g}{d a}=\frac{d+4}{a} g-2 \cdot \frac{2}{a} I
$$

$$
I=\int_{\mathbb{R}^{d}} d x \int_{0}^{\infty} d t_{1} d t_{2} e^{-\left(t_{1}+t_{2}\right)} t_{2} \int \mu_{0}\left(d b^{1}\right) \mu_{x}\left(d b^{2}\right) \chi\left[\operatorname{dist}\left(\omega_{\left[0, t_{1}\right]}^{1}, \omega_{\left[0, t_{2}\right]}^{2}\right) \leqq a\right] .
$$

The integration $\int_{0}^{\infty} d t_{2} e^{-t_{2}} t_{2} \ldots$ in (6.4) may be replaced by $\iint_{0}^{\infty} d s_{1} d s_{2} e^{-\left(s_{1}+s_{2}\right)} \ldots$ with $t_{2}=s_{1}+s_{2}$. For each $\left(s_{1}, s_{2}\right)$,we split the path

$$
\omega_{\left[0, t_{2}\right]}^{2}=\omega_{\left[0, s_{1}\right]}^{2} \cup \omega_{\left[s_{1}, s_{1}+s_{2}\right]}^{2} \text {. }
$$

For a specified $b\left(s_{1}\right)=y$ the two paths in the above splitting are independent. 
Furthermore, translation invariance permits us to replace $\int d x$ by $\int d y$. Thus:

$$
\begin{aligned}
I= & \int_{\mathbb{R}^{d}} d y \int_{0}^{\infty} d t_{1} e^{-t_{1}} \int_{0}^{\infty} d s_{1} d s_{2} e^{-\left(s_{1}+s_{2}\right)} \int \mu_{0}\left(d b^{1}\right) \mu_{y}\left(d b^{2}\right) \mu_{y}\left(d b^{3}\right) \\
& \cdot \chi\left[\operatorname{dist}\left(\omega_{\left[0, t_{1}\right]}^{1}, \omega_{\left[0, s_{1}\right]}^{2} \cup \omega_{\left[0, s_{2}\right]}^{3}\right) \leqq a\right]
\end{aligned}
$$

(where we also used a reflection in time, for $\omega^{2}$ ).

By the simplest case of the inclusion-exclusion principle,

$$
\begin{aligned}
\chi\left[\operatorname{dist}\left(\omega^{1}, \omega^{2} \cup \omega^{3}\right) \leqq a\right]= & \chi\left[\operatorname{dist}\left(\omega^{1}, \omega^{2}\right) \leqq a\right]+\chi\left[\operatorname{dist}\left(\omega^{1}, \omega^{3}\right) \leqq a\right] \\
& -\chi\left[\operatorname{dist}\left(\omega^{1}, \omega^{i}\right) \leqq a ; i=2 \text { and } 3\right] .
\end{aligned}
$$

Using it in (6.6), we get

$$
I=2 g-F(a),
$$

which, when combined with (6.3), yields the claimed (6.2)

The right-hand side of (6.1) provides us with an exact expression for $W(g(a))$. In order to derive the bounds (4.6) one has now to produce bounds on $F(a)$, in terms of $g(a)$. This is done next.

\section{b) The Lower Bound on $W(g)$}

The lower bound is quite natural. Since $F(a)$ represents events with two intersections (see Fig. 2) one may expect it to be related to the square of $g(a)$. The key tool for the following result is just the Schwarz inequality.

Lemma 6.2. In any dimension, for $a \leqq \alpha$ :

$$
F(a) \geqq c_{+}(\alpha) g(a)^{2}
$$

with a dimension dependent constant $c_{+}(\alpha)>0$ for which $c_{+}(0)$ is given in (4.8).

Proof. Let us denote by $\mathscr{C}_{i}$ the condition

$$
\sup _{0 \leqq s \leqq t_{i}} \pm \frac{x \cdot\left(b^{i}(s)-b^{i}(0)\right)}{|x|} \geqq \frac{|x|-\alpha}{2}, \text { with }+ \text { for } i=1, \text { and }- \text { for } i=2 .
$$

Clearly

$$
\begin{aligned}
F(a) \geqq & \int_{\mathbb{R}^{d}} d x \int_{0}^{\infty} d t_{1} e^{-t_{1}} \int \mu_{0}\left(d b^{1}\right) \chi\left[\mathscr{C}_{1}\right] \\
& \cdot\left\{\int_{0}^{\infty} d t_{2} e^{-t_{2}} \int \mu_{x}\left(d b^{2}\right) \chi\left[\operatorname{dist}\left(\omega_{\left[0, t_{1}\right]}^{1}, \omega_{\left[0, t_{2}\right]}^{2}\right) \leqq a\right]\right\}^{2},
\end{aligned}
$$

since without the characteristic function $\chi\left[\mathscr{C}_{1}\right]$ we have an equality. Applying the Schwarz inequality we obtain.

$$
\begin{aligned}
F(a) \geqq & \left(\int_{\mathbb{R}^{d}} d x \int_{0}^{\infty} d t_{1} e^{-t_{1}} \int \mu_{0}\left(d b^{1}\right) \chi\left[C_{1}\right]\right)^{-1} \\
& \cdot\left\{\int_{\mathbb{R}^{d}} d x \int_{0}^{\infty} d t_{1} e^{-t_{1}} \int d \mu_{0}\left(b^{1}\right) \int_{0}^{\infty} d t_{2} e^{-t_{2}} \int \mu_{x}\left(d b^{2}\right) \chi\left[\mathscr{C}_{1}\right]\right. \\
& \left.\cdot \chi\left[\operatorname{dist}\left(\omega_{\left[0, t_{1}\right]}^{1}, \omega_{\left[0, t_{2}\right]}^{2}\right) \leqq a\right]\right\}^{2} .
\end{aligned}
$$


By the symmetry of the integral in the brackets $\{-\}$, one may replace $\chi\left[\mathscr{C}_{1}\right]$ there with

$$
\frac{1}{2}\left(\chi\left[\mathscr{C}_{1}\right]+\chi\left[\mathscr{C}_{2}\right]\right) \geqq \frac{1}{2},
$$

the inequality being subject to the second constraint which is present in (6.11).

Without $\chi\left[\mathscr{C}_{1}\right]$, the bracketed expression is just $g$. Therefore we have derived (6.9), with the following value for $c_{+}(\alpha)$ :

$$
c_{+}(\alpha)^{-1}=4 \int_{\mathbb{R}^{d}} d x \int_{0}^{\infty} d t e^{-t} \int \mu_{0}(d x) \chi\left[\sup _{0 \leqq s \leqq t} \frac{x \cdot b(s)}{|x|} \geqq \frac{|x|}{2}\right] .
$$

To evaluate the integral, we use the reflection principle for the one dimensional projection of $b(s)$. One gets

$$
\begin{aligned}
c_{+}(\alpha)^{-1} & =2 \int_{\mathbb{R}^{d}} d x \int_{0}^{\infty} d t e^{-t} \int \mu_{0}(d x) \chi\left[\frac{x \cdot b(t)}{|x|} \geqq \frac{|x|-\alpha}{2}\right] \\
& =2 \int_{\mathbb{R}^{d}} d x \int_{0}^{\infty} d t e^{-t} \int_{|x-\alpha|+/ 2} d z \frac{1}{\sqrt{2 \pi t}} e^{-z^{2} /(2 t)}
\end{aligned}
$$

[using (2.1)]. The value of $c_{+}(0)$ is given in (4.7).

\section{c) The Upper Bound}

Lemma 6.3. For any $\delta>0$

$$
F(a) \leqq c_{\delta} g^{1+\frac{2}{d+2+\delta}}
$$

with the constant given in (4.8).

Although the above inequality completes the derivation of the qualitatively appealing picture described in Sects. 4 and 5 , it is less satisfactory than its counterpart (6.9). Note that any upper bound on $F(a)$ leads to a lower bound on $g(a)$. It turns out, however, that $g(a)$ may be bounded below by means of a more direct argument - with a better result for the critical dimension $d=4$ (see Sect. 7). For these reasons, the proof of (6.14) would be described here in a somewhat abbreviated form.

The main difficulty in bounding $F$ from above by const $g^{2}$ is that the two "intersections" required in $F(a)$ (see Fig. 2) can be highly correlated - if both occur in the vicinity of $x$. In fact, the contribution from the simplest of such events has the following consequence [see (7.22)]

$$
F(a) \geqq \frac{\text { const }}{a^{d-2}} \geqq \text { const } a^{(6-d)} g(a)^{2} .
$$

Hence $F(a) / g(a)^{2} \rightarrow \infty$, as $a \rightarrow 0$, in $d>6$ dimensions.

The above problem does not exist in estimating the quantity

$$
T(a)-\int_{\mathbb{R}^{d}} d x \int_{\mathbb{R}^{d}} d y T_{x, y}(a),
$$


where

$$
T_{x, y}(a)=\iiint_{0} \prod_{n=1}^{3}\left(d t_{n} e^{-t_{n}}\right) \int \mu_{0}\left(d b^{1}\right) \mu_{x}\left(d b^{2}\right) \mu_{y}\left(d b^{3}\right) \chi\left[\mathscr{D}_{2}\right] \chi\left[\mathscr{D}_{3}\right]
$$

with $\mathscr{D}_{i}$ denoting the events, $\operatorname{dist}\left(\omega_{\left[0, t_{1}\right]}^{1}, \omega_{\left[0, t_{i}\right]}^{i} \leqq a\right.$.

The event $\mathscr{D}_{2} \cap \mathscr{D}_{3}$ is the non-exclusive union of two sequences of events. In one, the trajectory of $b^{1}(t)$ comes to within distance $a$ from $\omega_{\left[0, t_{3}\right]}^{3}$ after reaching such a neighborhood of $\omega_{\left[0, t_{2}\right]}^{2}$. In the other sequence, the roles of $\omega^{2}$ and $\omega^{3}$ are reversed. By a simple stopping time argument, the contribution to $T(a)$ of each of these scenarios is exactly $g^{2}$. Thus one gets:

$$
\left(g(a)^{2} \leqq\right) T(a) \leqq 2 g(a)^{2} .
$$

Proof of Lemma 6.3. Returning to $F(a)$, one may combine the integrations over the two paths $b^{2}$ and $b^{3}$, in (6.2) into a single-path integral [reversing the step (6.5)], and a single time integral. This leads to the following representation

$$
\begin{aligned}
F(a)= & \int_{\mathbb{R}^{d}} d x \int_{0}^{\infty} d t_{1} e^{-t_{1}} \int_{0}^{\infty} d t e^{-t} \int \mu_{0}\left(d b^{1}\right) \mu_{x}\left(d b^{2}\right) \\
& \cdot \chi\left[\operatorname{dist}\left(\omega_{\left[0, t_{1}\right]}^{1}, \omega_{[0, t]}\right) \leqq a\right] \times\left(t_{\mathrm{L}}-t_{\mathrm{F}}\right),
\end{aligned}
$$

where $t_{\mathrm{F}}$ and $t_{\mathrm{L}}$ are the values of the first and the last times $s \in[0, t]$ at which $\operatorname{dist}\left(\omega_{\left[0, t_{1}\right]}^{1}, b(s)\right) \leqq a$.

Without the term $\left(t_{\mathrm{L}}-t_{\mathrm{F}}\right)$, the right-hand-side of $(6.18)$ would be just $g(a)$. By the Hölder inequality, we get for each $p>1$

$$
F(a) \leqq g(a)^{1-\frac{1}{p}} B(a)^{\frac{1}{p}}
$$

where $B(a)$ is the quantity obtained by replacing $\left(t_{\mathrm{L}}-t_{\mathrm{F}}\right)$ in $(6.25)$ with the factor:

$$
\left(t_{\mathrm{L}}-t_{\mathrm{F}}\right)^{p}=\mathrm{p}(\mathrm{p}-1) \int_{0}^{\infty} d u u^{p-2} \int_{0}^{\infty} d s \chi\left[s \geqq t_{F} ; s+u \leqq t_{\mathrm{L}}\right]
$$

Reversing the steps which led from (6.2) to (6.18), and using (in addition to the strong Markov property) Eq. (2.1), one gets

$$
B(a)=p(p-1) \int_{0}^{\infty} d u u^{p-2} e^{-u} \int_{\mathbb{R}^{d}} d x \int_{\mathbb{R}^{d}} d y e^{u \Delta}(x, y) T_{x, y}(a) .
$$

By (6.17), the uniform bound $e^{u \Delta}(x, y) \leqq(2 \pi u)^{-d / 2}$ [see (2.1)], and the choice of $p=1+(d+\delta) / 2$, we have

$$
B(a) \leqq\left[\frac{(d+\delta)(d+\delta+1)}{2(2 \pi)^{d / 2}} \Gamma\left(\frac{\delta}{2}\right)\right] g(a)^{2} .
$$

The substitution of (6.22) in (6.19) (with the above choice of $p$ ) results in the claimed bound (6.14) on $F(a)$.

\section{Direct Estimates of $F(a)$ and $g(a)$}

In this section we derive the bounds (4.7) on $F(a)$ (part 7c). It is also shown (in 7a and $7 \mathrm{~b}$ ) how $g(a)$ may be estimated by a more direct approach, which yields the 
leading behavior of $g(a)$ (in a qualitative sense) in all the cases except for the important logarithmic upper bound in $d=4$ dimensions (derived in Proposition 5.2). Note that the logarithmic lower bound for $d=4$, presented in $7 \mathrm{~b}$, is better than what we got via the a priori estimate of the $\beta$-function of Lemma 6.3 [in a remark after (5.12)].

The upper bounds of $7 \mathrm{a}$ and $7 \mathrm{~b}$ are derived by an adaptation of a specially simple (and quite versatile) argument of Spencer [5] for the proof of nonintersection in $d>4$ dimensions. The crux of it was an upper bound on the probability of intersection of the paths of lattice random walks, provided by the expected value of the size of their intersection. For the opposite bound, of $7 \mathrm{~b}$, we evaluate also the second moment, and use the Schwarz inequality.

The direct lower bound on $g(a)$ has also an alternative derivation, due to Sokal, which is given in [14]. I am grateful to Felder and Fröhlich for reminding me of the relevance of this estimate for the problem discussed here.

\section{a) An Upper Bound for $d>4$ (Following Spencer [5])}

The basic ingredient needed for the direct estimates is the exact expression for the ball-hitting probability (which has an easy derivation)

$$
\int_{0}^{\infty} d t \int \mu_{x}(d b) \chi\left[\operatorname{dist}\left(\omega_{[0, t]}, y\right) \leqq a\right]=\frac{G(x, y)}{G(0, a)}
$$

for $|x-y| \geqq a$, where $G(x, y)$ (the "free propagator") is the kernel, $G(x, y)$ $=(-\Delta+1)^{-1}(x, y)$, (and $G(0, a)$ stands for $G(0, z)$ with $\left.|z|=a\right)$.

The Fourier-transform of $G(0, x)$ is $\left(1+p^{2}\right)^{-1}$. We shall also use its following properties

i) $G(0, x) /\left[d|B(1)||x|^{2-d}\right] \leqq 1$, and $\rightarrow 1$ as $|x| \rightarrow 0$,

$|B(r)|$ being the volume of the ball of radius $r$, and

ii) $\int d x G(0, x)=1$.

Let us denote

$$
\int v\left(d b^{1} d b^{2}\right) \ldots=\int_{\mathbb{R}^{d}} d x \int_{0}^{\infty} d t_{1} e^{-t_{1}} \int_{0}^{\infty} d t_{2} e^{-t_{2}} \int \mu_{0}\left(d b^{1}\right) \mu_{x}\left(d b^{2}\right) \ldots
$$

and

$$
A_{i}=\omega_{\left[0, t_{i}\right]}^{i} .
$$

Thus $v$ is the measure over pairs of paths, with which

$$
g(a)=\int v\left(d b^{1} d b^{2}\right) \chi\left[\operatorname{dist}\left(A_{1}, A_{2}\right] \leqq a\right] .
$$

Trivial geometric considerations show that

$$
\chi\left[\left|V_{a / 2}\left(A_{1}, A_{2}\right)\right| \neq 0\right] \leqq \chi\left[\operatorname{dist}\left(A_{1}, A_{2}\right) \leqq a\right] \leqq \frac{\left|V_{2 a}\left(A_{1}, A_{2}\right)\right|}{|B(a)|},
$$

where $V_{r}\left(A_{1}, A_{2}\right)=\left\{z \in \mathbb{R}^{d} \mid \operatorname{dist}\left(A_{i}, z\right) \leqq r\right\}$, and $|V|$ is the $d$-dimensional volume of the set $V$. 
We shall now apply (7.7) - the main point being that while it is difficult to evaluate the integral (7.6), it is easy to evaluate the corresponding integral of $\left|V_{a}\right|$ (as well as to estimate its higher moments).

Proposition 7.1. In any dimension

$$
g(a) \leqq \hat{c} a^{d-4}(1+o(1))(\text { as } a \rightarrow 0)
$$

with $\hat{c}=\left[4^{2-d} d^{2}|B(1)|^{3}\right]^{-1}$.

Proof. Substituting (7.7) in (7.6) one obtains

$$
\begin{aligned}
g(a) & \leqq \int_{\mathbb{R}^{d}} d z \int v\left(d b^{1} d b^{2}\right) \chi\left[z \in V_{2 a}\left(A_{1}, A_{2}\right)\right] / \mid B(a) \\
& =\int d z \int v\left(d b^{1} d b^{2}\right) \prod_{i=1}^{2} \chi\left[\operatorname{dist}\left(\omega_{\left[0, t_{i}\right]}^{i}, z\right) \leqq 2 a\right] /|B(a)| \\
& =|B(a)|^{-1} G(0,2 a)^{-2} \int_{\mathbb{R}^{d}} d x \int_{\mathbb{R}^{d}} d z G(0, z) G(0, x) \\
& =|B(a)|^{-1} G(0,2 a)^{-2},
\end{aligned}
$$

where use was made of (7.1) and (7.3). By (7.2), the last expression reduces to (7.8).

Thus the simple estimate (7.7) leads to a rather good bound for $d>4$, although the above result does not, by itself, convey any information about $d<4$, and not much about the critical dimension $d=4$.

\section{b) Direct Lower Bounds for $g(a)$}

Using the left-hand side of (7.7), and then the Schwartz inequality we obtain the following lower bound for $g(a)$.

$$
g(a) \geqq \int v\left(d b^{1} d b^{2}\right) \chi\left[\left|V_{a / 2}\left(A_{1}, A_{2}\right)\right| \neq 0\right] \geqq \frac{\left(\int v\left(d b^{1} d b^{2}\right)\left|V_{a / 2}\left(A_{1}, A_{2}\right)\right|\right)^{2}}{\int v\left(d b^{1} d b^{2}\right)\left|V_{a / 2}\left(A_{1}, A_{2}\right)\right|^{2}} .
$$

Evaluating the moments of $\left|V_{a / 2}\right|$ one gets:

Proposition 7.2. In any dimension

$$
g(a) \geqq\left(4 \int_{|u| \geqq a / 2} d u G(0, u)^{2}+4^{2(d-2)} \hat{c} a^{4-d}\right)^{-1}
$$

(with $\hat{c}$ of (7.8)) and hence, for $a \leqq 1$,

$$
g(a) \geqq \begin{cases}C(d-4) a^{d-4}, & d>4, \\ c[1+|\ln a|]^{-1}, & d=4, \\ \left(\frac{4}{(2 \pi)^{d}} \int_{\mathbb{R}^{d}} d p\left(1+p^{2}\right)^{-2}\right)^{-1}=C(4-d), & d<4 .\end{cases}
$$

Proof. To estimate the denominator in (7.10) we write:

$$
\int v\left(d b^{1} d b^{2}\right)\left|V_{a / 2}\left(A_{1}, A_{2}\right)\right|^{2}=\int_{\mathbb{R}^{d}} d u \int_{\mathbb{R}^{d}} d v \int v\left(d b^{1} d b^{2}\right) \chi\left[u, v \in V_{a / 2}\left(A_{1}, A_{2}\right)\right] .
$$

There are two possibilities for the order in which the neighborhoods of $u$ and $v$ can be reached by each of the paths $b^{i}(t)$. Splitting the paths in a natural way, i.e. 
conditioning on the site of the first hit, one easily obtains:

$$
\begin{aligned}
& \int v\left(d b^{1} d b^{2}\right)\left|V_{a / 2}\left(A_{1}, A_{2}\right)\right|^{2}=\iint d u d v \int v\left(d b^{1} d b^{2}\right) \chi\left[u, v \in V_{a / 2}\left(A_{1}, A_{2}\right)\right] \\
& \quad \leqq 2 \iiint \int_{|y-z| \geqq a / 2} d x d y d z G(0, y) G(y, z)[G(x, y) G(y, z)+G(x, z) G(z, y)] / G(0, a / 2)^{2} \\
& \quad+\iiint_{|u-v| \leqq a} d x d u d v G(0, u) G(x, u) / G(0, a / 2)^{2} \\
& \quad=4 \int_{|z|>a / 2} d z G(0, z)^{2} / G(0, a / 2)^{4}+|B(1)| a^{d} / G(0, a)^{2} .
\end{aligned}
$$

The numerator in (7.10) equals $\left[G(0, a / 2)^{-2}\right]^{2}$, by the argument of (7.9). The substitution of (7.14) (with (7.2)) in (7.10) results therefore in the claimed bound (7.11). The transition to (7.12) can be made using the Fourier transform of $G(0, x)$, which is $\left(1+p^{2}\right)^{-1}$.

\section{c) Direct Estimates of $F(a)$}

The method used in 7a yields rather directly the following upper bound for the quantity $F(a)$, defined by (6.2):

$$
\begin{aligned}
F(a) \leqq & 2 \int_{\mathbb{R}^{d}} d x \iint_{|u| \geqq a} d u d v G(0, u) G(0, v) G(x, u) G(u, v)\left[|B(a)| G(0,2 a)^{2}\right]^{-2} \\
& +\int_{\mathbb{R}^{d}} d x G(0, x) G(0,2 a)^{-1} \\
& \leqq \\
c & \left.c(a) a^{2(d-4)}+\hat{c}|B(1)| a^{d-2}\right](1+o(1)),
\end{aligned}
$$

where

$$
\nabla(a)=\iint_{|u|>a} d u d v G(0, u) G(u, v) G(v, 0) \cong \begin{cases}C \frac{1}{6-d} a^{6-d}, & d>6, \\ c o n s t|\ln a|, & d=6, \\ \int_{\mathbb{R}^{d}} d p\left(1+p^{2}\right)^{-3}, & d<6,\end{cases}
$$

as $a \rightarrow 0$.

Therefore

$$
F(a) \leqq C a^{2(d-4)+\min (0,6-d)}\left\{|6-d|^{-1} \chi[d \neq 6]+c|\ln a| \chi[d=6]\right\} .
$$

For an opposite bound, let us first make the trivial observation that by (7.1) (with $y=0$ )

$$
F(a) \geqq \int d x G(0, x) / G(0, a) \geqq[d|B(1)|]^{-1} a^{d-2} .
$$

Combining it with (6.9) we have

$$
F(a) \geqq\left[\frac{1}{2} d|B(1)|\right]^{-1} a^{d-2}+\frac{1}{2} c_{+} g(a)^{2},
$$

and thus, using (7.12),

$$
F(a) \geqq C a^{2(d-4)+\min (0,6-d)}\left\{|d-4|^{2} \chi[d \neq 4]+c(1+|\ln a|)^{-1} \chi[d=4]\right\} .
$$

These results are summarized in (4.7) of Proposition 4.1 and used in Sect. 5. 


\section{Summary and Open Problems}

We have seen in Sect. 5 that the "renormalization group" equation described by Proposition 4.1 is an effective tool for the study of the intersection properties of Brownian motion. It would be interesting to see refinements of these techniques, e.g. to resolve the issue mentioned next. An even more important development would be an extension of these methods to some non-Markovian cases. Following are some specific problems.

i) Find the exact behavior, in $d<4$ dimensions, of the quantity

$$
\kappa(a)=\int_{0}^{\infty} d t_{1} e^{-t_{1}} \int_{0}^{\infty} d t_{2} e^{-t_{2}} \operatorname{Prob}\left(\operatorname{dist}\left(\omega_{\left[0, t_{1}\right]}^{1}, \omega_{\left[0, t_{2}\right]}^{2}\right) \geqq a \mid 0, x(a)\right),
$$

in which $x(a)$ scales, as $a \rightarrow 0$, with $|x(a)|=2 a$.

We join [4], where such a quantity is reconsidered for the random walks (and an error of [3] is corrected), with the conjecture that for $d=4$

$$
\kappa(a) \cong \frac{c}{\sqrt{\ln a^{-1}}}, \text { as } a \rightarrow 0 .
$$

Such a quantity plays an important role in the physical models which are mentioned in iii).

ii) The probability that Brownian paths come to within distance $a$ is of course analogous, and related to, the probability of an actual intersection of random walks on a lattice of spacing $a$. Considering the lattice random walks, it is natural to look at the ensemble, $\Omega_{x}$, of all the paths $\omega$ on $\mathbb{Z}^{d}$, with a given starting point $x$ and the weight $e^{\beta|\omega|}$, where $|\omega|$ is the number of steps. The normalizing factor and the mean square length are

$$
S=\sum_{\omega \in \Omega_{0}} e^{\beta|\omega|}, \quad \xi^{2}=\sum_{\omega \in \Omega_{0}}|x(\omega)|^{2} e^{\beta|\omega|} / S,
$$

with $x(\omega)=$ the last site visited by $\omega$.

For simple random walks $S$ and $\xi$ diverge at the critical point $\beta_{c}=\ln (2 d)^{-1}$. The following quantity

$$
\hat{g}(\beta)=\sum_{\substack{x \in \mathbb{Z}^{d} \omega^{1} \in \Omega_{0} \\ \omega^{2} \in \Omega_{0}}} e^{\beta\left|\omega_{1}\right|} e^{\beta\left|\omega_{2}\right|} \chi\left[\omega^{1} \cap \omega^{2} \neq \emptyset\right] /\left[S^{2} \xi^{d}\right]
$$

is analogous to $g(a)$, with $a=\sqrt{\beta_{c}-\beta} \cong 1 / \xi$.

Our methods are easily adaptable to the study of the limiting behavior of $\hat{g}(\beta)$, as $\beta>\beta_{c}$, with results which are given by (2.7), with the above correspondence. The problem is to study the analog of $\hat{g}(\beta)$ for self avoiding walks. E.g., prove there that

$$
\hat{g} \rightarrow 0 \quad \text { as } \quad \xi \rightarrow \infty \quad \text { (i.e. when } \beta \nearrow \beta_{c} \text { ), in } d \geqq 4 \text { dimensions. }
$$

iii) Derive non-perturbative bounds on the $\beta$-function of the $\lambda \phi_{d}^{4}$ field theory and ferromagnetic spin systems. The renormalized coupling constant in these theories admits a random walk representation which is similar to (8.4), albeit with weights which are more complicated than just $e^{\beta|\omega|}$. Nevertheless, the analysis of the critical behavior in these models has already been greatly aided by the intuition gathered from the study of the Brownian motion [8-10, 15]. (The physical significance of the quantity $\kappa(a)$ for such models is described in [9-12].) 

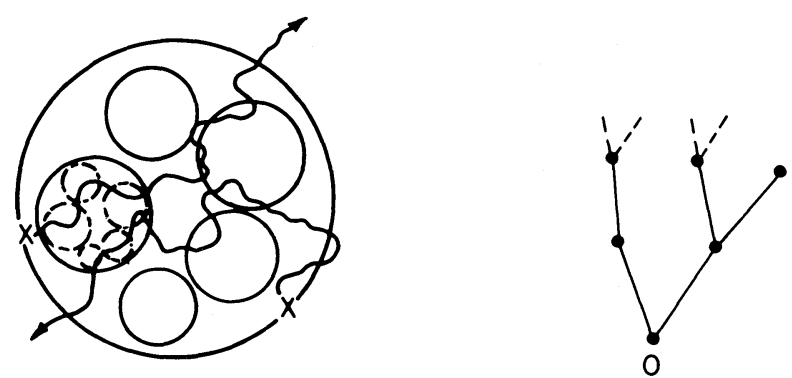

Fig. 3. A random-tree formulation of the intersection problem. (After [9])

\section{Appendix: A Percolation Formulation of the Intersection of Paths}

Another approach to the question of intersection of paths was presented in [9]. While it has not yet been useful for any complete argument, it offers yet another point of view. We shall provide here the proof of the main assertion which was made in the appendix of that work.

Since the probability of intersection of the paths of two independent Brownian motions depends only mildly on their starting points (being either zero or nonzero for all $x_{1} \neq x_{2}$ ), it may be studied by means of the following quantity.

$$
P=\int_{S} \sigma(d x) \int_{S} \sigma(d y) \operatorname{Prob}\left(\omega^{1} \cap \omega^{2} \cap B \neq \emptyset \mid 0, x\right) /\left(\int_{S} \sigma(d x)\right)^{2},
$$

where $B$ is the unit ball in $\mathbb{R}^{d}$, and $\sigma(d x)$ is the surface measure on $S^{d}=\partial B . P$ is therefore the probability that two paths which start independently on the boundary of a unit ball, intersect inside.

Let $Q_{1}=\left\{q_{1, j}\right\}_{j \in \mathbb{Z}_{+}}$be a partition of the unit ball $B$ into a family of balls, $q_{1, j}$ $C B$, whose interiors are disjoint, and which exhaust the volume of $B$, i.e. for which

$$
\sum_{j=1}^{\infty}\left|q_{1, j}\right|=|B| \text {. }
$$

Subpartitioning each of the balls of $Q_{1}$, and iteratively repeating this construction, one obtains a hierarchy of partitions which has the structure of a tree, whose "ascending" paths correspond to points of $B$. The correspondence is $1-1$, up to a set of measure zero (the "cheese") which may be ignored. For each pair of paths $\omega^{1}, \omega^{2}$ we now mark those points on the tree which correspond to balls (of various generations) which are hit by both paths. The marked points, and the edges which connect them form a random tree. The problem of paths intersection is thus reformulated as one of "percolation" (see Fig. 3, or the more detailed description in [9]).

A classical result states that for trees whose branching numbers are independent and identically distributed, the answer to the main question is determined by the single quantity $E\left(N_{1}\right)$, which is the expected value of the number of ascending links from the first vertex (and hence from each vertex which is reached). Specifically, the probability of "percolation" does not vanish if and only if $E\left(N_{1}\right) \geqq 1$ (unless $N_{1}$ $\equiv 1)$. In the case obtained here there are subtle correlations between the branching numbers. Consequently, we are not able to solve the problem in this form. 
Nevertheless, we would like to point out the following striking property of the Brownian motion.

Proposition A.1. Let $N_{1}\left(\omega^{1}, \omega^{2}\right)$ be the number of balls of $Q_{1}$ which are hit by both paths, and let $E(-)$ represent the average with respect to the probability measure

Then

$$
\sigma(d x) \sigma(d y) \mu_{x}\left(d b^{2}\right) \mu_{y}\left(d b^{2}\right) /\left(\int_{S} \sigma(d z)\right)^{2} .
$$

$$
E\left(N_{1}\left(\omega^{1}, \omega^{2}\right)\right) \text { is }\left\{\begin{array}{lll}
<1 & \text { if } & d>4 \\
=1 & & d=4 \\
>1 & & d<4
\end{array}\right.
$$

for every (nontrivial) partition $Q_{1}$ of $B$ into balls $\left\{q_{1, j}\right\}_{j \in \mathbb{Z}_{+}}$. Furthermore, the conditional distribution of the number of the next generation balls in $q_{k, j}$ which are intersected by both $\omega^{1}$ and $\omega^{2}$-conditioned on $q_{k, j}$ being intersected, has the same distribution as $N_{1}\left(\omega_{1}, \omega_{2}\right)$.

Thus, were we to ignore the existing interactions between the numbers of lines which are drawn at the various stages of our construction, we would obtain a solvable model. This "approximation," and Proposition A.1. offer another simple insight (but not yet a proof) into why

$$
P \neq 0 \text { if and only if } d<4 \text {. }
$$

Proof. Consider a path which starts at $z$, with $|z|>1$. The probability of it intersecting the unit ball $B$ is eactly $|z|^{2-d}$. If the intersection does occur then the site of the "first hit" of $B$ has a slightly $a$-symmetric distribution, which tends to $\sigma(d x) / \int_{S^{d}} \sigma(d u)$ when $|z| \rightarrow \infty$. Thus, by the renewal property of the Brownian motion:

$$
\begin{aligned}
P= & \lim _{|z| \rightarrow \infty} \int \mu_{z}\left(d b^{1}\right) \mu_{z}\left(d b^{2}\right) \chi\left[\omega^{1} \cap \omega^{2} \cap B \neq \emptyset\right] / \int \mu_{z}\left(d b^{1}\right) \\
& \cdot \mu_{z}\left(d b^{2}\right) \chi\left[\omega^{i} \cap B \neq \emptyset ; i=1,2\right]
\end{aligned}
$$

and, similarly,

$$
\begin{aligned}
E\left(N_{1}\left(\omega^{1}, \omega^{2}\right)\right)= & \lim _{z \rightarrow \infty} \sum_{j=1}^{\infty} \operatorname{Prob}\left(\omega^{i} \cap q_{1, j} \neq \emptyset ; i=1,2 \mid z, z\right) / \\
& \cdot \operatorname{Prob}\left(\omega^{i} \cap B \neq \emptyset ; i=1,2 \mid z, z\right) .
\end{aligned}
$$

Let $\left(x_{j}, r_{j}\right)$ be the centers, and radia, of the balls $q_{1, j}$. Then, by (A.6) and the formula mentioned above

$$
\begin{aligned}
E\left(N_{1}\right) & =\lim _{z \rightarrow \infty} \sum_{j=1}^{\infty}\left(\frac{r_{i}}{\left|r_{i}-z\right|}\right)^{2(d-2)} /\left(\frac{1}{|z|}\right)^{2(d-2)} \\
& =\frac{1}{|B(1)|} \sum_{j=1}^{\infty}\left|q_{1, j}\right| r_{j}^{d-4} .
\end{aligned}
$$

Equation (A.3) follows now from the fact that $\sum_{j=1}^{\infty}\left|q_{1, j}\right|=|B(1)|$, and $r_{j}$ are less than 1. The above argument implies also the other statement made in the proposition. 
Remark. Instead of using balls in Proposition A.1 one could also take any $A \subset \mathbb{R}^{d}$ which is the closure of an open bounded subset of $\mathbb{R}^{d}$. For such a set, $\sigma(d x)$ should be replaced with the unique measure which minimizes the capacity integral:

$$
C(A)^{-1}=\inf \left\{\int_{A} \varrho(d x) \int_{A} \varrho(d y) \frac{1}{|x-y|^{d-2}} \mid \int_{A} \varrho(d x)=1\right\} .
$$

The general result is that if one partitions $A_{1}$ into sets which áre similar to $A_{2}$, then

$$
\left(\frac{\hat{C}\left(A_{1}\right)}{\hat{C}\left(A_{2}\right)}\right)^{2} E\left(N_{1}\right)=\sum_{j=1}^{\infty}\left(\frac{\left|q_{1, j}\right|}{\left|A_{1}\right|}\right)^{1+\left(1-\frac{4}{d}\right)} \text { which is } \begin{cases}<1 & \mathrm{~d}>4 \\ =1 & \mathrm{~d}=4 \\ >1 & \mathrm{~d}<4\end{cases}
$$

where $\hat{C}\left(A_{i}\right)=C\left(A_{i}\right) /\left|A_{i}\right|$. In particular, (A.3) holds in the general self similar case.

The derivation of (A.9) rests on the above argument and the facts that

i) the hitting probability, for large $z$, behaves as $\frac{C(A)}{z^{d-2}}$, and

ii) for similar sets $q$ and $A: C(q)=C(A)\left(\frac{|q|}{|A|}\right)^{(d-2) / d}$.

Note. After the submission of this article I learned that a $\beta$-function approach to the problems discussed here has been advocated by J. Fröhlich [13]. A closely related work of Felder and Fröhlich [14] appears in this issue. I am grateful to T. Hara, T. Hattori, and H. Tasaki for pointing out that Lemma 6.2 requires a restriction on $a$.

\section{References}

1. Kakutani, S.: On Brownian motions in $n$-space. Proc. Acad. Tokyo 20, 648 (1944)

2. Dvoretzky, A., Erdös, P., Kakutani, S.: Double points of Brownian paths in $n$-space. Acta Sci. Math. Szeged 12, 75 (1950)

3. Erdös, P., Taylor, S.J.: Some intersection properties of random paths. Acta Math. Acad. Sci. Hung. 11, 231 (1960)

4. Lawler, G.F.: The probability of intersection of Random walks in four dimensions. Commun. Math. Phys. 86, 539 (1982)

5. Spencer, T.: Private communication

6. Symanzik, K.: Small distance behavior in field theory and power counting. Commun. Math. Phys. 18, 227 (1970)

Callan, C.G. Jr.: Broken scale invariance in scalar field theory. Phys. Rev. D 2, 1541 (1970) Wilson, K.G.: Anomalous dimension and the breakdown of scale invariance in perturbation theory. Phys. Rev. D 2, 1478 (1970)

7. Bréżin, E., Le Guillou, J.C., Zinn-Justin, J.: In: Phase transitions and critical phenomena. Domb, C., Green, M.S., (eds.). London, New York, San Francisco: Academic Press 1976 Itzykson, C., Zuber, J.B.: Quantum field theory. New York: McGraw-Hill 1980

8. Symanzik, K.: Euclidean quantum field theory. I. Equations for a scalar model. J. Math. Phys. 7, $510(1966)$

9. Aizenman, M.: Geometric analysis of $\phi^{4}$ fields and Ising models. Commun. Math. Phys. 86, 1 (1982)

10. Fröhlich, J.: On the triviality of $\lambda \phi_{d}^{4}$ theories and the approach to the critical point in $d>4$ dimensions. Nucl. Phys. B 200, [FS4] 281 (1982)

11. Aragão de Carvalho, C., Caraciollo, S., Fröhlich, J.: Polymers and $g|\phi|^{4}$ theory in four dimensions. Nucl. Phys. B 215 [FS7], 209 (1983) 
12. Aizenman, M., Graham, R.: On the renormalized coupling constant and the susceptibility in $\phi_{4}^{4}$ field theory and the Ising model in four dimensions. Nucl. Phys. B 225 [FS9], 261 (1983)

13. Fröhlich, J.: Quantum field theory in terms of Random walks and Random surfaces. Cargèse (1983) lecture notes

14. Felder, G., Fröhlich, J.: Intersection properties of simple Random walks: a renormalization group approach. Commun. Math. Phys. 97, 111-124 (1984)

15. Aizenman, M.: Rigorous studies of critical behavior, to appear in the proceedings of the VIII sitges conference. L. Garrido (ed.), Berlin, Heidelberg, New York: Springer 1984

Communicated by A. Jaffe

Received January 9, 1984; in revised form April 23, 1984 